\title{
Morbilidades del neurodesarrollo asociadas con el nacimiento pretérmino con peso s1500 gramos entre 1993 y 2011 en España: estudio de una muestra de 1200 casos
}

\author{
Neurodevelopmental morbidities associated with preterm birth with a \\ weight of $\leq 1500$ grams between 1993 and 2011 in Spain: study of a \\ sample of 1200 cases
}

\begin{abstract}
Resumen
Objetivos: el parto muy prematuro puede originar discapacidades en múltiples dominios del desarrollo. Este artículo describe las morbilidades del neurodesarrollo que, según sus progenitores, presenta una muestra de 1202 niños/as nacidos/as con $\leq 1500$ gramos entre 1993 y 2011 en España y destaca algunas de las variables que influyen en ellas. Métodos: encuesta multi-método realizada entre abril 2013 y junio 2014 con soporte CATI y CAWI. Previamente, se realizó un extenso trabajo de campo cualitativo. Resultados: el $44,2 \%$ de la muestra presenta morbilidades ( $\bar{x} 1,78$ morbilidades por niño). Las más prevalentes son los trastornos del aprendizaje (34,4\% de los casos) y el trastorno por déficit de atención e hiperactividad $(31,5 \%)$. En este período, se produce un incremento relativo de morbilidades temporales frente a permanentes. Conclusiones: el porcentaje total de niños con morbilidades disminuye moderadamente entre 1993 y 2011. Las variables biológicas y médicas explicarían mejor que las familiares la presencia de morbilidades en esta población.
\end{abstract}

\section{Palabras clave}

Neurodesarrollo con muy bajo peso, desarrollo niños muy prematuros, nacidos con muy bajo peso, recién nacidos con $<1500 \mathrm{~g}$, niños muy prematuros, variables familiares en prematuridad, sociología de la prematuridad.

\begin{abstract}
Objectives: very preterm birth can cause disabilities in numerous developmental domains. This article describes the morbidities neurodevelopmental that, according to their parents, is present in a sample of 1202 children born with $\leq 1500$ grams between 1993 and 2011 in Spain and highlights some of the variables that influence them. Methods: Multi-method survey conducted with CATI and CAWI support between April 2013 and June 2014. Previously, extensive qualitative field work was carried out. Results: A total of $44.2 \%$ of the sample presents morbidities ( $\bar{x} 1.78$ morbidities per child). The most prevalent types are learning disorders (34.4\%) and attention deficit hyperactivity disorder $(31.5 \%)$. In this period, there is a relative increase in temporary versus permanent morbidities. Conclusions: The total percentage of children with morbidities decreased moderately between 1993 and 2011. The biological and medical variables would explain better than the family variables the presence of morbidities in this population.
\end{abstract}

\section{Keywords}

Neurodevelopment of very low weight infants, very low weight infant development, very low weight preterm birth, newborns of birth weight less than 1500 , very premature infants, family variables in prematurity, sociology of prematurity.

\section{Concepción Gómez Esteban \\ <conchagomez@cps.ucm.es>}

Universidad Complutense de Madrid. España

\section{Juan J. Sánchez Carrión $<j$ jsc@cps.ucm.es>}

Universidad Complutense de Madrid. España

\section{Fernando J. García Selgas \\ $<$ fgselgas@cps.ucm.es>}

Universidad Complutense de Madrid. España

\section{José M. Segovia Guisado <jmsegovi@ucm.es>}

Universidad Complutense de Madrid. España

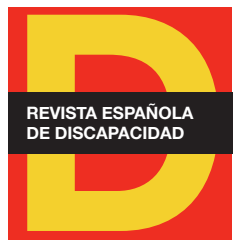

Para citar:

Gómez, C. et al. (2019): "Morbilidades del neurodesarrollo asociadas con el nacimiento pretérmino con peso $\leq 1500$ gramos entre 1993 y 2011 en España: estudio de una muestra de 1200 casos". Revista Española de Discapacidad, 7 (I): 29-47.

Doi: <https://doi.org/10.5569/23405104.07.01.02>

Fecha de recepción: 13-02-2018 Fecha de aceptación: 29-05-2019

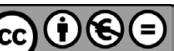




\section{Introducción ${ }^{1}$}

La prematuridad es una condición biológica que afecta a muchos de los factores que regulan el desarrollo. En general, los problemas de salud que pueden presentar los nacidos prematuramente son inversamente proporcionales al peso y a la edad gestacional (en adelante, EG) con los que nacen, aunque para todos los nacidos pretérmino hay un gradiente de riesgo, incluidos los nacidos a término temprano ( 37 y 38 semanas de $E G)$.

La inmadurez biológica de los bebés de muy bajo peso al nacer (MBPN), que son los nacidos con $<1500$ gramos, es el principal origen de las morbilidades que pueden aparecer en cualquier momento durante su desarrollo, incluso en la edad adulta (Costeloe et al., 2012; Darlow et al., 2013; Hille et al., 2007; Larroque et al., 2008; Saigal y Doyle, 2008; Shah et al., 2016; Taylor et al., 2009, entre otros); de ahí la importancia de prevenir el parto prematuro y de que estos niños tengan un adecuado seguimiento sociosanitario y educativo, que permita minimizar la eventual aparición de secuelas y su gravedad, tal y como reclaman muchos expertos (Dall'Oglio et al., 2010; Taylor, 2015) y las asociaciones de padres (Gómez, 2014).

Entre 1993 y 2011, en España los casos de bebés de MBPN aumentaron un $123 \%$ (Instituto Nacional de Estadística, 2013). Pese a este importante incremento, la tasa de supervivencia ha mejorado sustancialmente, especialmente desde 2005 (Guzmán et al., 2013; Hernández et al., 2005), aunque la morbilidad no ha disminuido en la misma medida, ni en España ni en otros países, especialmente en el grupo de prematuros extremos (los nacidos por debajo de la semana 28 de EG), como ponen de manifiesto los trabajos de Cheong et al., 2017; Moor et al., 2012 o Sánchez, 2014. Por ello, uno de los principales desafíos que enfrenta la neonatología actual es seguir disminuyendo la tasa de mortalidad de estos neonatos sin aumentar la morbilidad y la discapacidad y de ahí la necesidad de conocer a fondo la evolución de estos niños.

Este artículo pretende contribuir a este objetivo estudiando las morbilidades del neurodesarrollo más frecuentes en los niños nacidos con muy bajo peso en nuestro país y algunas de las variables que influyen en ellas, contando con una importante muestra de esta población.

Los resultados presentados proceden de un estudio transversal y correlacional sobre los nacidos con $\leq 1500$ en España entre 1993 y 2011, financiado por el Plan Nacional de I+D+i (ref. CSO2011-24294). Los objetivos de este estudio buscaban establecer una síntesis de los problemas bio-psico-sociales que presentan estos niños y analizar el impacto de las variables sociofamiliares en su desarrollo, conocer las principales consecuencias que tiene para las relaciones y prácticas familiares este tipo de nacimiento y estudiar los principales cambios y transformaciones producidos en la atención neonatal que se presta a estos niños y a sus familias.

\footnotetext{
1. Una versión previa de este artículo se ha publicado en septiembre de 2017 en la revista Global Pediatric Health. http://journals.sagepub.com/ doi/full/10.1177/2333794X17733372.

An English version of this article has been published in September 2017 in the journal Global Pediatric Health.

http://journals.sagepub.com/doi/full/10.1177/2333794X17733372.

Financiación: este proyecto de investigación ha sido financiado por el Plan Nacional de I+D+i (ref. CSO2011-24294).

Agradecimientos: A B. Sáenz-Rico de Santiago (UCM), M. Martín Carballo (UCA), A. Vicente Olmo (UCM), Mª J. Pascual Barragán y A. Coronado Marín (UCM) por su participación en diferentes etapas de este proyecto. Gracias también a la Sociedad Española de Neonatología, al Comité Español de Representantes de Personas con Discapacidad, a la Asociación de Padres de Niños Prematuros, a todos los hospitales, entidades y profesionales que atienden a los niños nacidos con muy bajo peso que han dado su apoyo a este proyecto y, muy especialmente, a la Dra. Sofía Salas.
} 
Estos amplios objetivos responden a nuestro interés por abrir una línea de trabajo en el campo de la sociología ${ }^{2}$ sobre un tema social que consideramos emergente: la gran prematuridad.

\section{Metodología}

Antes de realizar la encuesta, algunos de cuyos resultados vamos a presentar aquí, se realizó un análisis de datos secundarios y un amplio trabajo de campo cualitativo. Este trabajo incluyó observación no participante en algunas Unidades de Cuidados Intensivos Neonatales (UCIN) y la realización 4 grupos de discusión y 25 entrevistas en profundidad (tres grupos de discusión se realizaron con madres y padres y otro con personal de enfermería; por su parte, las entrevistas se hicieron a profesionales de todos perfiles implicados en la atención al desarrollo de los niños con MBPN y a familias, incluyendo también a adolescentes nacidos con $\leq 1500 \mathrm{~g}$ y a sus hermanos).

A partir de los resultados del trabajo cualitativo se elaboró un cuestionario de 48 preguntas autocumplimentado y estructurado -salvo la inclusión de 2 preguntas abiertas-, que se alojó en una página web. La población era la de los nacidos en España durante el período 1993-2011 con un peso $\leq 1500 \mathrm{~g}$ y la muestra obtenida finalmente fue de 1202 cuestionarios válidos (1190 tras ponderar los datos; véase anexo). La recolección de datos se realizó entre abril de 2013 y junio de 2014, mediante encuesta multi-método con tecnología CATI (Computer Assisted Telephone Interview) y CAWI (Computer Assisted Web Interview, creada para este estudio utilizando software de libre distribución).

A falta de un marco muestral del que generar una muestra probabilística, tuvimos que recurrir a una muestra intencional, con captación de los niños/familias por una variedad de vías y procedimientos que se explican a continuación y se resumen en el gráfico 1.

En primer lugar, se creó la página web del proyecto, que permitió posicionar un enlace en los buscadores de internet; de esta manera, cualquier persona que buscara un concepto relacionado con la prematuridad tenía la posibilidad de encontrar esa página y acceder al cuestionario que se colgó en ella.

En segundo lugar, se consiguió la colaboración desinteresada de hospitales y asociaciones, que enviaron el enlace a la encuesta por correo online y postal a las familias que tenían registradas en sus bases de datos, proporcionándoles también un número de teléfono y un email para contactar con miembros del grupo de investigación, que les ayudarían a cumplimentar la encuesta en caso de dificultad. Complementariamente se distribuyó un marcapáginas por diferentes centros de atención primaria, consultas hospitalarias privadas y

2. Algunos resultados de esta investigación se han presentado ya, entre otros, en los siguientes foros científicos: IX Encuentros de Teoría Sociológica (Madrid, 28-29 de junio de 2018); XXVI Congreso de Perinatología y Medicina Perinatal (Zaragoza, 27-28 de septiembre de 2017); XII Congreso Español de Sociología (Gijón, 30 de junio-2 de julio de 2016); XXVIII edición de los Cursos de Verano de la Universidad Complutense de Madrid (San Lorenzo de El Escorial, curso del 13 al 17 de julio de 2015); XXV Congreso de Perinatología y Medicina Perinatal (Sevilla, 20-22 de mayo de 2015); XXIV Congreso de Neonatología y Medicina Perinatal (Barcelona, 2-4 octubre 2013); XI Congreso Español de Sociología (Madrid, 10-12 de julio de 2013); VI Congreso Andaluz de Sociología (Cádiz, 28-29 de noviembre-1 de diciembre de 2012); VI Reunión Interdisciplinar de la red Genysi (Madrid, 15 de noviembre de 2012); también se presentarán resultados en el XXVII Congreso de Perinatología y Medicina Perinatal (Madrid, 2-4 de octubre de 2019). 
públicas, centros de atención temprana, etc. que contenía el enlace y un código $Q R$ que permitía el acceso a la encuesta online desde cualquier dispositivo.

Igualmente se realizó una difusión muy activa en las redes sociales, solicitando a diferentes asociaciones y colectivos relacionados con la prematuridad que distribuyeran en sus muros (Facebook) y entre sus contactos o grupos (Linkedin) un mensaje con el enlace al cuestionario. También se distribuyó el enlace en Twitter, con el hashtag \#menos1500. Para poder realizar esta tarea se creó una base de datos de más de 40 colectivos susceptibles de tener entre sus seguidores a familias con niños nacidos con peso $\leq 1500 \mathrm{~g}$.

El gráfico 1 muestra las distintas vías mediante las que se obtuvieron los 1202 cuestionarios.

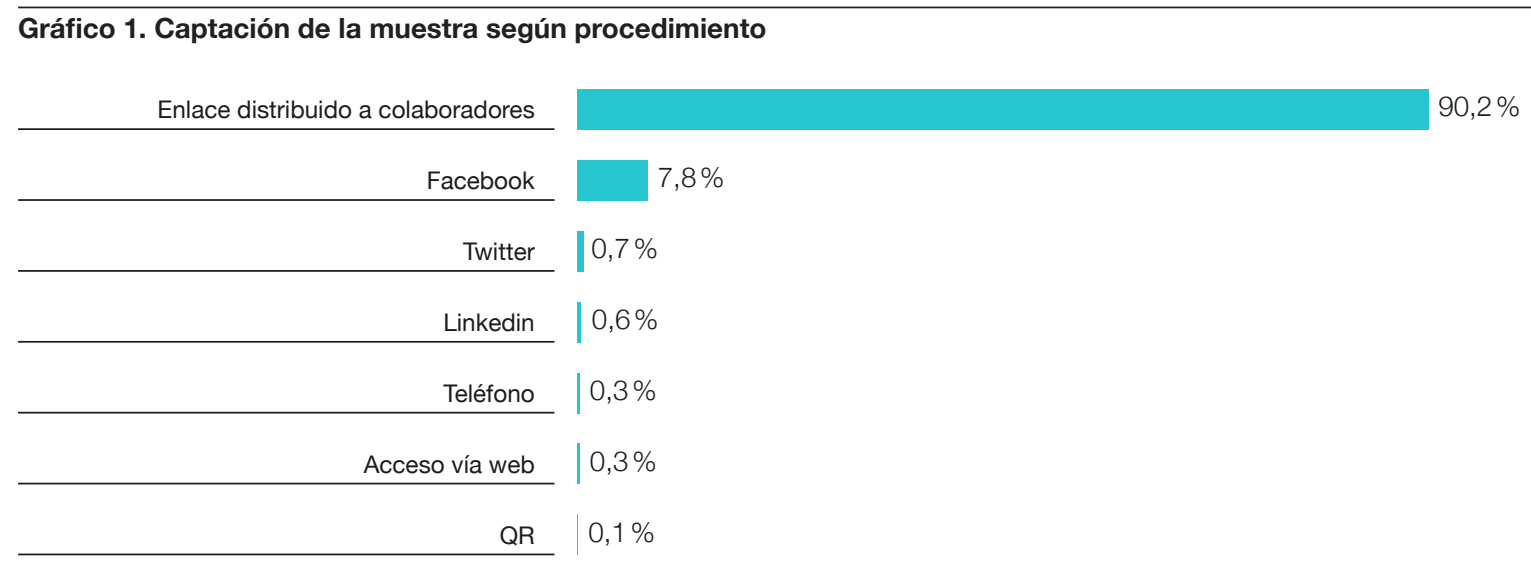

Fuente: elaboración propia.

Una vez obtenidos los datos, que se iban a analizar con el programa SPSSWIN, se detectó que en ellos había una sobrerrepresentación de nacidos pretérmino en los últimos años contemplados en nuestro estudio y una infrarrepresentación de los nacidos en los primeros. Para corregir ambas y hacer la muestra más representativa del conjunto de los niños nacidos con $\leq 1500 \mathrm{~g}$ entre 1993 y 2011 en España, esto es respecto al universo de referencia, se acudió a los datos proporcionados por el INE para 1993-2011 y, una vez comparados con ellos, se reequilibraron nuestros datos a partir de las dos variables principales, peso al nacer y año de nacimiento (aunque se consideró en su momento, no se reequilibró la muestra por edad gestacional puesto que el INE dispone de menos datos sobre esta variable que sobre peso, al menos en los primeros años del período establecido en nuestro estudio. Y, con respecto a la variable sexo, la diferencia entre los datos del INE y los nuestros era asumible; para una información más detallada sobre los datos obtenidos en la muestra y su ponderación, véase el anexo). Por último, cuando fue posible por existir datos, los resultados se compararon con los datos desagregados de 0-19 años de la Encuesta de Discapacidad, Autonomía personal y situaciones de Dependencia (INE, 2008), que es aún la principal referencia en España sobre limitaciones y discapacidades de la población general.

Algunas de las características más relevantes de la muestra obtenida son las siguientes: 


\begin{tabular}{|c|c|c|}
\hline Característica & Porcentaje/promedio & Observaciones \\
\hline Sexo: varones & $49,3 \%$ & \\
\hline Peso medio al nacimiento & $1152,2 \mathrm{~g}$ & \\
\hline Peso al nacimiento $<1000 \mathrm{~g}$ & $25,5 \%$ & $\begin{array}{l}\text { - } 8 \% \text { con }<750 \mathrm{~g} \\
\text { - } 17,5 \% \text { entre } 750-999 \mathrm{~g}\end{array}$ \\
\hline Edad gestacional (EG) media & 29,4 semanas & $\begin{array}{l}\text { - } 8,8 \% \text { de nacidos entre la semana } 22 \\
\text { y } 25\end{array}$ \\
\hline Neonatos nacidos con $\leq 1500 \mathrm{~g}$ y $>32 \mathrm{EG}$ & $19,7 \%$ & \\
\hline Tipo de parto & $\begin{array}{l}62,7 \% \text { único } \\
37,3 \% \text { múltiple }\end{array}$ & $\begin{array}{l}\text { - } 28,7 \% \text { gemelos } \\
\text { - } 8,6 \% \text { trillizos o más }\end{array}$ \\
\hline $\begin{array}{l}\text { Mortalidad en partos múltiples (hermano/s } \\
\text { fallecido/s antes del alta de la Unidad) }\end{array}$ & $13,4 \%$ & \\
\hline $\begin{array}{l}\text { Tiempo medio de estancia en Unidad de } \\
\text { Cuidados Intensivos Infantil (UCIN) }\end{array}$ & 36,4 días & \\
\hline $\begin{array}{l}\text { Titularidad del hospital donde estuvo } \\
\text { ingresado el neonato }\end{array}$ & 91,4 \% pública & $\begin{array}{l}\text { - } 6,3 \% \text { privada } \\
\text { - } 2,3 \% \text { gestión mixta (pública-privada) }\end{array}$ \\
\hline Edad media de las madres & 32,0 años & $\begin{array}{l}-1,07 \%<18 \text { años } \\
\text { - } 2,55 \%>40 \text { años }\end{array}$ \\
\hline $\begin{array}{l}\text { Tratamiento de infertilidad y/o de reproducción } \\
\text { asistida previo al embarazo }\end{array}$ & $24,2 \%$ & $\begin{array}{l}\text { - De estos, el 77,08\% fue fecundación } \\
\text { in vitro }\end{array}$ \\
\hline Madres nacidas en España & $94,3 \%$ & $\begin{array}{l}\text { - } 95 \% \text { de las parejas también habían } \\
\text { nacido en España }\end{array}$ \\
\hline $\begin{array}{l}\text { Acceso libre a la Unidad de Neonatología } \\
\text { durante } 24 \mathrm{~h} \text {. }\end{array}$ & $19,2 \%$ & - $51,5 \%$ en período $2009-2011$ \\
\hline Lactancia materna & $60,4 \%$ & - $82,4 \%$ en período $2009-2011$ \\
\hline $\begin{array}{l}\text { Posibilidad de practicar el método canguro y } \\
\text { piel con piel durante el ingreso del hijo }\end{array}$ & $64,5 \%$ & - $92,3 \%$ en período $2009-2011$ \\
\hline $\begin{array}{l}\text { Participación del hijo en algún/os programa/s } \\
\text { de seguimiento tras alta de la Unidad }\end{array}$ & $84 \%$ & \\
\hline $\begin{array}{l}\text { Tratamientos de estimulación y/o rehabilitación } \\
\text { recibidos }\end{array}$ & $63,1 \%$ & $\begin{array}{l}\text { - } 39,9 \% \text { tratamiento de estimulación } \\
\text { - } 23,2 \% \text { tratamiento de rehabilitación }\end{array}$ \\
\hline $\begin{array}{l}\text { Reingreso hospitalario tras el alta (todas las } \\
\text { causas) }\end{array}$ & $45,2 \%$ & $\begin{array}{l}\text { - } 23,9 \% \text { por problemas respiratorios } \\
\text { - } 15 \% \text { por algún tipo de operación } \\
\text { quirúrgica } \\
\text { - } 15,9 \% \text { por otros motivos }\end{array}$ \\
\hline Niños escolarizados & $92,4 \%$ & $\begin{array}{l}\text { - De estos, } 86 \% \text { por modalidad } \\
\text { ordinaria } \\
\text { - } 5,3 \% \text { por integración } \\
\text { - } 7,2 \% \text { por necesidades educativas } \\
\text { - } 1,5 \% \text { no sabe }\end{array}$ \\
\hline
\end{tabular}

Fuente: elaboración propia. 


\section{Descripción y cuantificación de morbilidades en niños de muy bajo peso al nacer}

El nacimiento muy prematuro se asocia con discapacidades en múltiples dominios del desarrollo (Van Baar et al., 2005). Para conocer las morbilidades que presentan los nacidos con $\leq 1500$ gramos entre 1993-2011, hemos preguntado a las familias qué morbilidades, principalmente del neurodesarrollo, presentaban sus hijos en el momento de contestar la encuesta, según los diagnósticos profesionales y los tratamientos que estaban recibiendo ${ }^{3}$.

En nuestro estudio, el porcentaje total de niños con morbilidades es del 44,2\%, muy superior al que presenta en España la población total de esa edad (solo tienen morbilidades el 1,9\%, según los datos de INE, 2008), siendo el promedio de morbilidades de 1,788 por niño. Un 8,6\% del total presenta solo morbilidades temporales, un $16,3 \%$ permanentes, un $10,3 \%$ tiene morbilidades de ambos tipos y el $12 \%$ tiene algún tipo de morbilidad no incluida en la clasificación que aplicamos en nuestro estudio.

Los datos recogidos en la tabla 2 ponen de manifiesto que los tipos más frecuentes de morbilidad en esta población aparecen tardíamente en el desarrollo. Son morbilidades de alta prevalencia como los trastornos del aprendizaje $(34,4 \%)$ y el trastorno por déficit de atención e hiperactividad (TDAH) $(31,5 \%)$, lo que concuerda grosso modo con los hallazgos de Aarnoudse-Moens et al., 2009; Bracewell et al., 2008; Larroque et al., 2008, entre otros. Se trata de trastornos considerados a veces poco severos pero que pueden afectar enormemente a los resultados académicos, la conducta y el desarrollo emocional de estos niños y, por tanto, a su futuro y al de sus familias.

Detengámonos en la parálisis cerebral infantil $(\mathrm{PCl})$, a menudo una morbilidad de graves consecuencias, que habría disminuido en este colectivo en los últimos años (Platt et al., 2007) o se habría mantenido estable pese al gran aumento producido en la supervivencia de los bebés más prematuros (Van Naarden et al., 2016). Según la tabla 2, la PCI habría disminuido, aunque no significativamente, si bien nuestros datos no permiten discernir qué tipo o tipos de parálisis serían los que habrían disminuido en el período analizado.

3. La clasificación de morbilidades utilizada en la encuesta se estableció basándose en la experiencia clínica de dos psicopedagogas que han colaborado en el diagnóstico y el seguimiento a grandes prematuros en hospitales de referencia y en otros centros; estas profesionales participaron en algunas fases del proyecto de investigación presentado aquí. 


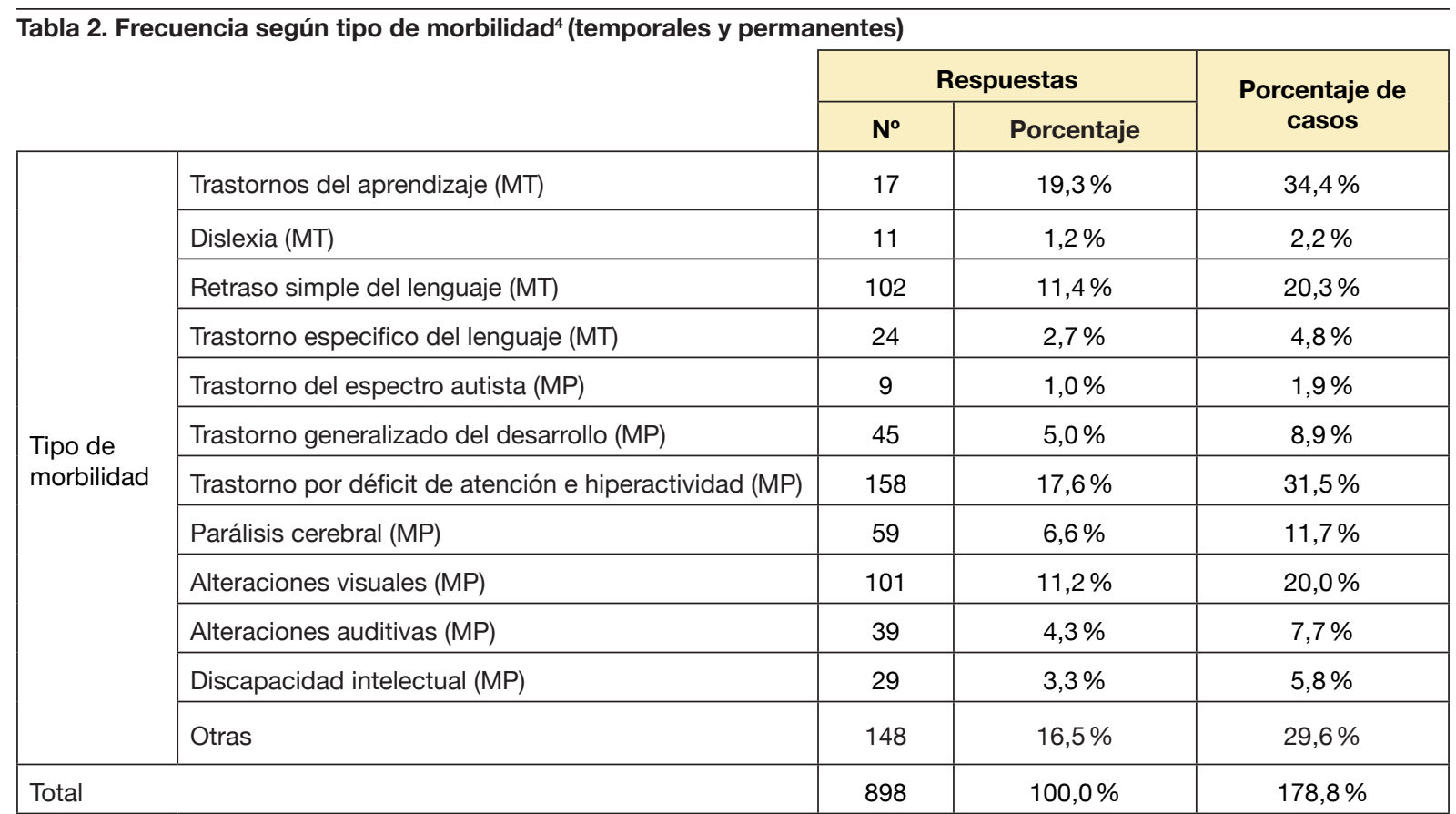

MT: morbilidades temporales MP: morbilidades permanentes Fuente: elaboración propia.

En nuestro estudio partíamos de la hipótesis de que a partir de 1998-1999 se habrían producido en España cambios esenciales para la supervivencia y mejora de la salud de los nacidos con $\leq 1500 \mathrm{~g}$. Por una parte, estos cambios se deberían al uso de nuevos tratamientos como corticoides prenatales, surfactante, optimización de la nutrición, CPAP (Continuous Positive Airway Pressure), etc., y a la introducción desde 2004 del llamado 'modelo de cuidados centrados en el desarrollo', que comienza a ser el modelo dominante a partir de 2009 en los hospitales públicos de nuestro país y, por otra parte, a la implantación de un seguimiento médico protocolizado de todos los casos de niños de MBPN, especialmente en los hospitales públicos (lo cual no quiere decir que tras el alta de la Unidad todos los niños se hayan beneficiado de programas de seguimiento médico y, sobre todo, de otros programas fundamentales para el desarrollo de los niños de muy bajo peso al nacer como son los de estimulación y/o rehabilitación. La cobertura pública de algunos de estos programas es claramente insuficiente en muchas comunidad autónomas, lo que obliga a un gran número de familias a hacer un esfuerzo económico importante para contratar de forma privada estos servicios y poder atender lo más tempranamente posible y durante todo el tiempo que la situación lo exija las necesidades de este tipo que tienen sus hijos). La falta de cobertura de estos programas, y a veces de información adecuada sobre los riesgos que tiene esta población y/o sobre los programas de prevención y atención existentes, así como otros factores de tipo sanitario y familiar contribuyen a explicar por qué el $16 \%$ de los niños de nuestra muestra no ha participado en ningún programa de apoyo al desarrollo.

4. Hay que señalar que en el cuestionario diseñado para la encuesta no se utilizó el término "morbilidades", poco conocido fuera del ámbito sanitario, sino el de "secuelas" para hacer más clara la pregunta a las familias. Si en este artículo hablamos de "morbilidades" es porque entendemos que metodológicamente no cabe hablar de "secuelas" si no hay un grupo de control, pese a que en muchos estudios que tampoco lo tienen se utilice este último término. 
Para verificar nuestra hipótesis sobre el efecto favorable que habrían tenido en la salud de los niños de MBPN los cambios en el cuidado y en la atención producidos entre 1993 y 2011, analizamos si había una relación entre el año de nacimiento de estos niños y la morbilidad que presentaban.

\begin{tabular}{|c|c|c|c|c|c|c|}
\hline & & \multicolumn{4}{|c|}{ Año de nacimiento del niño } & \multirow{2}{*}{ Total } \\
\hline & & 1993-1998 & $1999-2003$ & 2004-2008 & 2009-2011 & \\
\hline \multirow{2}{*}{$\begin{array}{l}\text { Niños con } \\
\text { morbilidad/es }\end{array}$} & $\%$ en este período & $52,7 \%$ & $41,6 \%$ & $44,0 \%$ & $38,9 \%$ & $44,2 \%$ \\
\hline & Recuento & 237 & 281 & 375 & 244 & 1137 \\
\hline
\end{tabular}

Fuente: elaboración propia.

\begin{tabular}{|c|c|c|c|c|c|c|c|c|}
\hline & & 1993-1998 & $1999-2003$ & 2004-2008 & 2009-2011 & Total & Rho & Sig $^{\mathrm{a}}$ \\
\hline \multirow{13}{*}{$\begin{array}{l}\text { Tipo } \\
\text { de } \\
\text { morbilidad }\end{array}$} & Trastornos del aprendizaje & $20,5 \%$ & $20,0 \%$ & $12,6 \%$ & $8,5 \%$ & 173 &,- 139 & ,000 \\
\hline & Dislexia & $3,5 \%$ &, $7 \%$ &, $1 \%$ &, $0 \%$ & 11 &,- 117 & ,000, \\
\hline & Retraso simple del lenguaje & $6,7 \%$ & $2,3 \%$ & $9,6 \%$ & $18,1 \%$ & 102 & ,145 & 000, \\
\hline & Trastorno específico del lenguaje & $2,0 \%$ & $2,4 \%$ & $1,7 \%$ & $2,5 \%$ & 24 &,- 006 & ,845 \\
\hline & Trastorno del espectro autista & $2,2 \%$ & ,6\% & ,2\% & ,7\% & 9 &,- 049 & ,093 \\
\hline & $\begin{array}{l}\text { Trastorno generalizado del } \\
\text { desarrollo }\end{array}$ & $6,7 \%$ & $2,4 \%$ & $2,8 \%$ & $4,9 \%$ & 45 &,- 030 & ,293 \\
\hline & $\begin{array}{l}\text { Trastorno por déficit de atención } \\
\text { e hiperactividad }\end{array}$ & $24,0 \%$ & $15,2 \%$ & $10,9 \%$ & $7,1 \%$ & 158 &,- 173 & ,000 \\
\hline & Parálisis cerebral & $6,0 \%$ & $5,3 \%$ & $5,0 \%$ & $4,5 \%$ & 59 &,- 028 & ,343 \\
\hline & Alteraciones visuales & $12,0 \%$ & $8,2 \%$ & $9,2 \%$ & $6,0 \%$ & 101 &,- 063 & ,029 \\
\hline & Alteraciones auditivas & $4,6 \%$ & $2,5 \%$ & $3,8 \%$ & $2,8 \%$ & 39 &,- 024 & ,402 \\
\hline & Discapacidad intelectual & $6,3 \%$ & $2,7 \%$ & $1,0 \%$ & $1,2 \%$ & 29 &,- 112 & ,000 \\
\hline & Otras & $12,4 \%$ & $12,7 \%$ & $13,4 \%$ & $13,3 \%$ & 148 &,- 017 &, 566 \\
\hline & Sin morbilidades & $47,2 \%$ & $58,3 \%$ & $56,1 \%$ & $61,0 \%$ & 636 & ,076 & ,010 \\
\hline \multicolumn{2}{|l|}{ Total } & 237 & 281 & 375 & 245 & 1138 & & \\
\hline
\end{tabular}

Fuente: elaboración propia.

La tabla 3 recoge el porcentaje de morbilidades de esta población en función de la segmentación temporal establecida en nuestro estudio. Confirmando nuestra hipótesis, se puede observar que a medida que pasa el tiempo disminuye moderadamente el porcentaje total de niños con morbilidades del neurodesarrollo, si bien 
conviene ser prudentes en relación con los datos de la cohorte más joven (nacidos entre 2009 y 2011) dada la dificultad para poder establecer antes de los 3 o 4 años un diagnóstico certero de las morbilidades que son más prevalentes en esta población ${ }^{5}$.

La tabla 4 presenta la evolución de cada morbilidad estudiada en nuestra investigación.

A partir de estos y otros datos de nuestro estudio, podemos señalar que:

- $\quad$ En general, los trastornos de salud disminuyeron moderadamente entre 1993 y 2011 en la población de nacidos con $\leq 1500 \mathrm{~g}$ ( Tau-c de Kendall es -,077 y coeficiente de correlación de Spearman -,067), incluso, parece que esta ha sido la tendencia entre los nacidos con $<1000 \mathrm{~g}$, y ello pese al incremento producido en la tasa de supervivencia de los bebés más inmaduros (García-Muñoz et al., 2015, entre otros).

- Los trastornos del aprendizaje y el trastorno por déficit de atención e hiperactividad, las "discapacidades invisibles" (Doyle y Saigal, 2009), fueron las morbilidades más prevalentes en esta población ${ }^{6}$.

- $\quad$ En el conjunto del período estudiado, se observa un incremento relativo de las morbilidades temporales -cada vez mejor conocidas y, por tanto, más fáciles de diagnosticar- y una disminución de las permanentes, producida sin duda por los avances en la atención perinatal y el tratamiento médico terapéutico que reciben estos niños.

\section{Variables que influyen en la morbilidad}

Para conocer la influencia de las diferentes variables estudiadas en el proyecto elaboramos, a modo tentativo, un modelo causal de regresión logística tomando como variable dependiente la presencia/ausencia de morbilidad en el niño y analizando la influencia de tres tipos de variables, que llamamos, respectivamente, biológicas, médicas y de cuidados y sociofamiliares ${ }^{7}$.

Como se ha podido observar en la tabla 1, entre las variables biológicas analizadas están el sexo del neonato, el año de nacimiento, el peso al nacer, la EG, el tipo de embarazo y el uso materno de tratamientos de fertilidad y reproducción asistida.

Las variables médicas y de cuidados incluyen el tiempo que el neonato estuvo ingresado en la UCIN, el tiempo total de estancia en la Unidad de Neonatología (en adelante, UN), el tipo de titularidad del hospital de ingreso al nacimiento, las prácticas de atención centrada en la familia recibidas o realizadas durante la estancia del neonato en la UN, el posible seguimiento médico por nacimiento con peso $<1500 \mathrm{~g}$, la existencia o

5. En relación con lo dicho, señalar que el alto porcentaje de retraso simple del lenguaje de los nacidos entre 2009 y 2011 que aparece en la tabla 4 sin duda expresa el desfase madurativo frecuente en los grandes prematuros.

6. Añadir a esta información que, de los niños de la muestra escolarizados, el 41,3\% ha necesitado algún tipo de apoyo/s. De estos últimos, el $56 \%$ ha necesitado profesor de apoyo en el centro, el 42,2\% refuerzo educativo, el 18,6\% adaptación curricular, el 22,7\% rehabilitación y/o fisioterapia, el $51,3 \%$ atención logopédica y el $25 \%$ apoyo psicológico.

7. En el análisis logístico se excluyeron variables que, como el tiempo de estancia en la UCIN, los reingresos hospitalarios o la necesidad de rehabilitación, podrían no ser independientes de morbilidades previas, por ejemplo, de morbilidades de tipo congénito. 
no de recursos sanitarios especializados en la localidad de residencia familiar, los programas y tratamientos de estimulación temprana y rehabilitación recibidos por el bebé, reingresos hospitalarios, etc.

Entre las variables sociofamiliares se cuentan, entre otras, el número total de hijos de la familia, cuántos han nacido prematuramente, cuántos con $\leq 1500 \mathrm{~g}$., la edad de los/as progenitores/as, su nacionalidad, su estado civil, su nivel educativo y su situación laboral, el tamaño de la localidad de residencia familiar, el total de ingresos económicos de la unidad familiar, los recursos y apoyo disponibles para la familia, los problemas anímicos experimentados por el/la cuidador/a principal, etc.

El resultado más notable del análisis (tabla 5) es que la mayoría de las variables analizadas en este estudio tienen poco peso para explicar la presencia/ausencia de morbilidades del neurodesarrollo en esta población. Estos resultados son acordes con el enfoque no clínico de nuestro estudio, que no ha recogido ni analizado datos específicos sobre la prevalencia de patologías que tienen un efecto importante sobre la morbilidad y su evolución en esta población (tales como las hemorragias peri e intraventriculares, la displasia broncopulmonar, la retinopatía de la prematuridad, etc.) ni sobre deficiencias congénitas y problemas del parto, que, en conjunto, explicarían el $57 \%$ de las deficiencias en menores de 20 años (INE, 2008). Sin embargo, esto no significa que las variables analizadas en nuestro estudio no ejerzan ninguna influencia.

Tabla 5. Influencia de variables en la presencia/ausencia de morbilidades
Modelo causal de regresión logística
(Variable dependiente presencia/ausencia de morbilidades)
\begin{tabular}{|l|c|c|c|c|c|c|c|c|}
\hline Variables & B & E.T. & Wald & gl & Sig. & Exp(B) & \multicolumn{1}{l}{ I.C. 95\% para EXP(B) } \\
\hline Inferior & Superior \\
\hline P5_a_01(1) Sexo masculino &, 913 &, 188 & 23,616 & 1 & .000 & 2,492 & 1,724 & 3,602 \\
\hline P6_a Año de nacimiento &,- 039 &, 017 & 5,160 & 1 & .023 &, 961 &, 929 &, 995 \\
\hline P8_a_2 Peso al nacer &,- 002 &, 000 & 24,492 & 1 & .000 &, 998 &, 998 &, 999 \\
\hline $\begin{array}{l}\text { P13_a_01(1) Madre no nacida en } \\
\text { España }\end{array}$ & 1,181 &, 416 & 8,071 & 1 & .004 & 3,258 & 1,442 & 7,359 \\
\hline $\begin{array}{l}\text { P15_a_01(1) Nacimiento hospital } \\
\text { privado }\end{array}$ & 1,175 &, 450 & 6,820 & 1 & .009 & 3,240 & 1,341 & 7,828 \\
\hline P27_a_1 Malestar anímico cuidador/a &, 071 &, 028 & 6,552 & 1 & .010 & 1,073 & 1,017 & 1,133 \\
\hline $\begin{array}{l}\text { P27_a_10 Escasez recursos } \\
\text { sanitarios especializados en localidad } \\
\text { de residencia }\end{array}$ &, 067 &, 027 & 6,264 & 1 & .012 & 1,069 & 1,015 & 1,127 \\
\hline Constante & 1,168 &, 485 & 5,806 & 1 & .016 & 3,214 & & \\
\hline
\end{tabular}

Nota: en este análisis logístico se han incluido todas las variables recogidas en nuestra encuesta que resultaron de mayor influencia en análisis previos.

El modelo explica el 20,5\% (R-cuadrado de Nagelkerke) del hecho de tener morbilidades, clasifica correctamente el 66,2\% de los casos, tiene una sensibilidad del $59,4 \%$ y una especificidad del $71,9 \%$.

Fuente: elaboración propia. 
En relación con las variables biológicas, nuestro modelo muestra que los niños que han nacido con menor peso, menor EG y hace más tiempo tienen un mayor riesgo de morbilidad (relaciones estadísticamente significativas). El peso al nacer (como en Conrad et al., 2010) y el sexo masculino (Gäddlin et al., 2008; García et al., 2013; Raiser et al., 2014) son también en nuestro estudio las dos variables biológicas más relevantes de las estudiadas.

De las variables médicas y de cuidados, el tipo de titularidad del hospital de ingreso al nacimiento, y más en concreto la titularidad privada del mismo, tiene un gran peso para explicar las morbilidades. Aun considerando que nuestra muestra de niños de hospitales privados es pequeña, hay que destacar que el $43 \%$ de los nacidos en hospitales públicos tuvo secuelas, frente al $63,4 \%$ de los nacidos en hospitales privados (también según nuestros datos, en los hospitales privados se realizan más cesáreas y los niños nacidos en este tipo de hospitales han participado menos en programas de seguimiento del desarrollo).

Dos variables socio-familiares destacan en el análisis. La primera, es el malestar anímico que padece el/la cuidador/a principal una vez que el bebé es dado de alta de la UN, un factor influyente en la morbilidad de los niños de MBPN como también señalan los pediatras españoles (Domínguez et al., 2016). Otros estudios ponen de manifiesto cómo en el caso de los recién nacidos prematuros, el desarrollo cognitivo y los resultados conductuales correlacionan negativamente con la depresión y la ansiedad materna (McManus y Poehlmann, 2012). La segunda variable relevante es el hecho de que la madre no haya nacido en España y sea migrante. En nuestra muestra el mayor número de mujeres no nacidas en nuestro país proviene de Centroamérica y Sudamérica ( $2.9 \%$ del total). Señalar también que la probabilidad de morbilidades del neurodesarrollo en los niños de MBPN aumenta si el/la otro/a progenitor/a tampoco ha nacido en España.

\section{Discusión}

Esta última cuestión plantea un tema interesante para el debate. Varios estudios han mostrado que la mayoría de las mujeres migrantes que viven en España son sanas, tienen una menor incidencia de prácticas de riesgo durante la gestación y que sus hijos, comparados con los de las madres nacidas en España, tienen mejor peso al nacer; y estas circunstancias se producen pese a que muchas de esas madres provienen de contextos socioeconómicamente más desfavorecidos y suelen experimentar desventajas sociales en los países de acogida.

Aunque para explicar la morbilidad en esta población, sin duda se pueden considerar otros factores como el mayor riesgo de defectos congénitos en la población migrante, pensamos que los factores que mejor explican la morbilidad de estos niños están relacionados con lo que sucede después del nacimiento y, sobre todo, con el escaso apoyo, privado o público ${ }^{8}$, que reciben en España las familias que tienen hijos nacidos con muy bajo peso para enfrentar de manera adecuada la compleja y costosa crianza de estos infantes. Lógicamente,

8. De las familias con hijos nacidos con $\leq 1500 \mathrm{~g}$ entre 1993 y 2011 en España, el $31 \%$ de las familias encabezadas por padres que habían nacido en España y el $63 \%$ de las encabezadas por padres que eran migrantes no recibieron ningún tipo de apoyo durante los primeros meses de la vida del hijo, ni siquiera de sus familiares más cercanos. 
la escasez de apoyos tiene un mayor impacto cuando las circunstancias sociofamiliares son más precarias -ya sea por desventajas sociales, bajo nivel de ingresos ${ }^{9}$ o educativo- y dificulta seriamente el poder conciliar las obligaciones familiares $y / o$ laborales con el cuidado y el seguimiento sociosanitario y educativo requerido por estos niños (entre otros, Drotar et al. (2006) han señalado la necesidad de proporcionar apoyo a las familias para minimizar los problemas de salud relacionados con el peso muy bajo al nacer y otros factores de riesgo asociados a esta condición biológica).

Si para finalizar atendemos a cuestiones de orden metodológico, es necesario reconocer aquí que somos conscientes de que puede haber un cierto grado de parcialidad en nuestros resultados debido a la estructura de la muestra, que está basada en la participación voluntaria de familias cuyos hijos han sido atendidos, mayoritariamente, en hospitales de referencia, que son los centros donde se trata a los niños de MBPN más gravemente afectados; de hecho, en términos relativos, nuestra muestra tiene un mayor porcentaje de varones -que tienen más riesgo- y de bebés con menos EG que la población de niños de muy bajo peso nacidos en España entre 1993 y 2011 si consideramos los datos del INE (aunque estos datos son muy incompletos para los primeros años del período que hemos estudiado). Además, más de un $20 \%$ de nuestra muestra son adolescentes de entre 15 y 19 años, una cuestión relevante dado que los problemas de salud y el deterioro de las funciones neurocognitivas en niños de MBPN pueden aumentar con la edad (O'Brien et al., 2004). Los dos aspectos señalados ayudarian a explicar por qué, según nuestros datos, los factores socio-familiares parecen tener poca influencia en la incidencia de morbilidad en esta población, mientras que las variables relacionadas con el tratamiento y los cuidados médicos tienen un peso considerable.

Con vistas a mejorar el potencial explicativo de este tipo de estudios, queremos señalar la necesidad de que se cree un marco muestral de la población de niños prematuros a partir no solo de los datos del INE sino de los datos del Grupo SEN1500 de la Sociedad Española de Neonatología y de otros registros, que permita obtener una muestra probabilística, al menos para una parte de este período (2002-2011). También la necesidad de que el diseño de este tipo de estudios sea longitudinal, con el fin de controlar tanto el efecto de la memoria/recuerdo en las respuestas de los entrevistados como el orden temporal de los hechos y que se incluyan variables propiamente clínicas en el cuestionario, dado el impacto que parecen tener sobre las morbilidades del neurodesarrollo en individuos con MBPN.

Para finalizar, destacar el apoyo efectivo que nos han proporcionado los tres entes promotores observadores de este estudio (Cermi, APREM y SENeo) y un importante número de hospitales, profesionales, asociaciones y familias. Sin duda, ello ha permitido obtener un cuantioso material, en especial, una gran muestra de este tipo de población, que está compuesta por niños de todas las comunidades autónomas., lo que permite analizar muchos aspectos de su desarrollo biopsicosocial, los factores contextuales que influyen en él y el impacto que tiene el muy bajo peso al nacer en el conjunto de la vida familiar, aunque muchas de estas cuestiones no se hayan tratado aquí.

Para finalizar, señalar algo que nos parece de vital importancia: que este estudio ha detectado algunos de los factores que permiten conocer qué nacidos con $\leq 1500 \mathrm{~g}$ son "doblemente de riesgo" (Guralnick, 2012), lo que puede ayudar al diseño de programas sociosanitarios y educativos para atender mejor las necesidades de los niños más vulnerables de este colectivo.

9. Nuestros resultados han puesto de relieve también que, aunque con menor significación, la escasez de ingresos económicos del hogar es otra de las variables que influyen en el desarrollo de morbilidades en esta población (por ejemplo, Brooks-Gunn, 2003, ya señaló que los ingresos familiares bajos y el estado de pobreza eran mejores predictores de las puntuaciones obtenidas al medir el cociente intelectual en los niños prematuros de 5 años que la educación materna u otros factores sociofamiliares y así sucede también en nuestro caso). 
Aarnoudse-Moens, C. S. et al. (2009): Meta-analysis of neurobehavioral outcomes in very preterm and/or very low birth weight children. Pediatrics, 124 (2): 717-728 (en línea). <https://doi.org/10.1542/peds.2008-2816>, acceso 3 de junio de 2019.

Bracewell, M. A. et al. (2008): The EPICure study: growth and blood pressure at 6 years of age following extremely preterm birth. Archives of Disease in Childhood. Fetal and Neonatal Edition, 93 (2): 108-114 (en línea). <https:// doi.org/10.1136/adc.2007.118596>, acceso 3 de junio de 2019.

Brooks-Gunn, J. (2003): Do you believe in magic? What can we expect from early childhood intervention programs. Social Policy Reports, 17 (1): 3-14.

Cheong, J. L. Y. et al. (2017): Changing neurodevelopment at 8 years in children born extremely preterm since the 1990 s. Pediatrics, 139 (6): e20164086 (en línea). <https://doi.org/10.1542/peds.2016-4086>, acceso 3 de junio de 2019.

Conrad, A. L. et al. (2010): Biological and enviromental predictors of behavioral sequelae in children born preterm. Pediatrics, 125 (1): 83-89 (en línea). <https://doi.org/10.1542/peds.2009-0634>, acceso 3 de junio de 2019.

Costeloe, K. L. et al. (2012): Short term outcomes after extreme preterm birth in England: comparison of two birth cohorts in 1995 and 2006 (the EPICure studies). British Medical Journal, 345: e7976 (en línea). <https://doi. org/10.1136/bmj.e7976>, acceso 3 de junio de 2019.

Dall'Oglio, A. M. et al. (2010): Do healthy preterm children need neuropsychological follow-up? Preschool outcomes compared with term peers. Developmental Medicine \& Child Neurology 52 (10): 955-961 (en línea). <https://doi. org/10.1111/j.1469-8749.2010.03730.x>, acceso 3 de junio de 2019.

Darlow, B. A. et al. (2013): Psychosocial outcomes of young adults born very low birth weight. Pediatrics, 132 (6): 1521-1528 (en línea). <https://doi.org/10.1542/peds.2013-2024>, acceso 3 de junio de 2019.

Domínguez, B. et al. (2016): Seguimiento en las consultas de Pediatría de Atención Primaria de los grandes prematuros: nacidos con menos de 32 semanas de gestación o menos de 1500 gramos. Revista de Pediatría de Atención Primaria, 18: 311-316.

Doyle, L. D. y Saigal, S. (2009): Long-term outcomes of very preterm or tiny infants. NeoReviews, 10 (3): $130-37$ (en línea). <https://doi.org/10.1542/neo.10-3-e130>, acceso 3 de junio de 2019.

Drotar, D. et al. (2006): The impact of extremely low birth weight on the families of school-aged children. Pediatrics, 117 (6): 2006-2013 (en línea). <https://doi.org/10.1542/peds.2005-2118>, acceso 3 de junio de 2019.

Gäddlin P. O. et al. (2008): A fifteen-year follow-up of neurological conditions in VLBW children without overt disability: Relation to gender, neonatal risk factors, and end stage MRI findings. Early Human Development, 84 (5): 343-349 (en línea). <https://doi.org/10.1016/j.earlhumdev.2007.09.013>, acceso 3 de junio de 2019.

García, P. et al. (2013): Evolución a los 2 años de edad corregida de una cohorte de recién nacidos con peso inferior o igual a $1.500 \mathrm{~g}$ de los hospitales pertenecientes a la red neonatal SEN1500. Anales de Pediatría, 79: 279-287 (en línea). <https://doi.org/10.1016/j.anpedi.2013.03.017>, acceso 3 de junio de 2019.

García-Muñoz, F. et al. (2015): Changes in perinatal care and outcomes in newborns at the limit of viability in Spain: The EPI-SEN study. Neonatology, 107 (2): 120-129 (en línea). <https://doi.org/10.1159/000368881>, acceso 3 de junio de 2019. 
Gómez, C. (2014): Asociaciones de padres y seguimiento multidisciplinar de la gran prematuridad. Revista Española de Discapacidad, 2 (1): 203-213 (en línea). <https://doi.org/10.5569/2340-5104.02.01.12>, acceso 3 de junio de 2019.

Guralnick, M. (2012): Preventive interventions for preterm children: effectiveness and developmental mechanisms. Journal of Developmental \& Behavioral Pediatrics, 33: 352-364.

Guzmán, J. M. et al. (2013): Estudio comparativo de morbimortalidad de los RN incluidos en la Red SEN1500 durante el período (2006-2010) (en línea). <http://www.se-neonatal.es/Portals/O/SEN-1500/Morbi-Morta_2006_2010sen1500.pdf>, acceso 14 de abril de 2015.

Hernández, N. et al. (2005): Morbilidad a los dos años en niños con un peso al nacimiento menor de 1500 g. Anales de Pediatría, 62 (4): 320-327.

Hille, E. T. et al. (2007): Functional outcomes and participation in young adulthood for very preterm and very low birth weight infants: the Dutch Project on Preterm and Small for Gestational Age Infants at 19 years of age. Pediatrics, 120 (3): e587-595 (en línea). <https://doi.org/10.1542/peds.2006-2407>, acceso 3 de junio de 2019.

Instituto Nacional de Estadística (INE) (2013): Estadística del Movimiento natural de la población. Nacimientos (en línea). <http://www.ine.es/dyngs/INEbase/es/operacion.htm?c=Estadistica_C\&cid=1254736177007\&menu=ul tiDatos\&idp=1254735573002>, acceso 3 de junio de 2019.

Instituto Nacional de Estadística (INE) (2008): Encuesta de Discapacidad, Autonomía personal y situaciones de Dependencia (en línea). <http://www.ine.es/dyngs/INEbase/es/operacion.htm?c=Estadistica_C\&cid=1254736 176782\&menu=resultados\&secc=1254736194716\&idp=1254735573175>, acceso 20 de abril de 2015.

Larroque, B. et al. (2008): Neurodevelopmental disabilities and special care of 5-year-old children born before 33 weeks of gestation (the EPIPAGE study): a longitudinal cohort study. The Lancet, 371 (9615): 813-820 (en línea). <https://doi.org/10.1016/S0140-6736(08)60380-3>, acceso 3 de junio de 2019.

McManus, B. M. y Poehlmann, J. (2012): Maternal depression and perceived social support as predictors of cognitive function trajectories during the first 3 years of life for preterm infants in Wisconsin. Child: Care, Health and Development, 38 (3): 425-434 (en línea). <https://doi.org/10.1111/j.1365-2214.2011.01253.x>, acceso 3 de junio de 2019.

Moor, T. et al. (2012): Neurological and developmental outcome in extremely preterm children born in England en 1995 and 2006: the EPICure studies. British Medical Journal, 345: e7961 (en línea). <https://doi.org/10.1136/ bmj.e7961>, acceso 3 de junio de 2019.

O'Brien, F. et al. (2004): The neurodevelopmental progress of infants less than 33 weeks into adolescence. Archives of Disease in Childhood, 89 (3): 207-211 (en línea). <https://doi.org/10.1136/adc.2002.006676>, acceso 3 de junio de 2019.

Platt, M. J. et al. (2007): Trends in cerebral palsy among infants of very low birthweight $(<1500 \mathrm{~g})$ or born prematurely (<32 weeks) in 16 European centres: a database study. The Lancet, 369 (9555): 43-50 (en línea). <https:// doi.org/10.1016/S0140-6736(07)60030-0>, acceso 3 de junio de 2019.

Raiser, E. et al. (2014): Readmission of preterm infants less than 32 weeks gestation into early childhood. Global Pediatric Health, 1: 1-6 (en línea). <https://doi.org/10.1177/2333794X14549621>, acceso 3 de junio de 2019.

Saigal, S. y Doyle, L. W. (2008): An overview of mortality and sequelae of preterm birth from infancy to adulthood. The Lancet, 371 (9608): 261-269 (en línea). <https://doi.org/10.1016/S0140-6736(08)60136-1>, acceso 3 de junio de 2019. 
Sánchez, M. (2014): Límite de viabilidad en la actualidad. Anales de Pediatría, 80 (6): 346-347 (en línea). <https:// doi.org/10.1016/j.anpedi.2014.01.019>, acceso 3 de junio de 2019.

Shah, P. S. et al. (2016): Neonatal outcomes of very low birth weight and very preterm neonates: An international comparison. The Journal of Pediatrics, 177: 144-152.e6 (en línea). <https://doi.org/10.1016/j.jpeds.2016.04.083>, acceso 3 de junio de 2019

Taylor, H. G. (2015): Multiple risks for long-term cognitive impairments following preterm birth. Acta Pædiatrica, 104 (3): 218-220 (en línea). <https://doi.org/10.1111/apa.12900>, acceso 3 de junio de 2019.

Taylor, N. M. et al. (2009): Differential vulnerability of global motion, global form, and biological motion processing in full-term and preterm children. Neuropsychologia, 47 (13): 2766-2778 (en línea). <https://doi.org/10.1016/j. neuropsychologia.2009.06.001>, acceso 3 de junio de 2019.

Van Baar, A. L. et al. (2005): Very preterm birth is associated with disabilities in multiple developmental domains. Journal of Pediatric Psychology, 30 (3): 247-255 (en línea). <https://doi.org/10.1093/jpepsy/jsi035>, acceso 3 de junio de 2019.

Van Naarden, K. L. et al. (2016): Birth prevalence of cerebral palsy: A population-based study, Pediatrics, 137 (1): 1-9 (en línea). <https://doi.org/10.1542/peds.2015-2872>, acceso 3 de junio de 2019. 
Anexo 1. Muestra y ponderación de los datos

1. Comparación de los datos de la muestra obtenida en la encuesta y los del INE

\begin{tabular}{|c|c|c|c|c|c|}
\hline \multicolumn{6}{|c|}{ AÑO DE NACIMIENTO DEL HIJO } \\
\hline & & Encuesta & Porcentaje & INE & Porcentaje \\
\hline \multirow{19}{*}{ Válidos } & 1993 & 29 & 2,4 & 2000 & 3,2 \\
\hline & 1994 & 23 & 1,9 & 2033 & 3,2 \\
\hline & 1995 & 22 & 1,8 & 2181 & 3,4 \\
\hline & 1996 & 23 & 1,9 & 2171 & 3,4 \\
\hline & 1997 & 20 & 1,7 & 2277 & 3,6 \\
\hline & 1998 & 34 & 2,8 & 2472 & 3,9 \\
\hline & 1999 & 33 & 2,7 & 2706 & 4,3 \\
\hline & 2000 & 53 & 4,4 & 2959 & 4,7 \\
\hline & 2001 & 48 & 4,0 & 3044 & 4,8 \\
\hline & 2002 & 72 & 6,0 & 3436 & 5,4 \\
\hline & 2003 & 64 & 5,3 & 3561 & 5,6 \\
\hline & 2004 & 73 & 6,1 & 3617 & 5,7 \\
\hline & 2005 & 57 & 4,7 & 3852 & 6,1 \\
\hline & 2006 & 38 & 3,2 & 3851 & 6,1 \\
\hline & 2007 & 66 & 5,5 & 4312 & 6,8 \\
\hline & 2008 & 98 & 8,2 & 4999 & 7,9 \\
\hline & 2009 & 88 & 7,3 & 4844 & 7,6 \\
\hline & 2010 & 140 & 11,6 & 4542 & 7,2 \\
\hline & 2011 & 221 & 18,4 & 4468 & 7,1 \\
\hline & Total & 1202 & 100,0 & 63325 & 100,0 \\
\hline
\end{tabular}




\begin{tabular}{|c|c|c|c|c|c|}
\hline \multicolumn{6}{|c|}{ SEXO } \\
\hline & & Encuesta & Porcentaje & INE & Porcentaje \\
\hline \multirow{3}{*}{ Válidos } & Niño & 592 & 49,4 & 32088 & 50,7 \\
\hline & Niña & 607 & 50,6 & 31237 & 49,3 \\
\hline & Total & 1199 & & & \\
\hline Perdidos & Sistema & 3 & & & \\
\hline \multicolumn{2}{|l|}{ Total } & 1202 & 100,0 & 63325 & 100,0 \\
\hline
\end{tabular}

\begin{tabular}{|c|c|c|c|c|c|}
\hline \multicolumn{6}{|c|}{ PESO AL NACER } \\
\hline & & Encuesta & Porcentaje & INE & Porcentaje \\
\hline \multirow{6}{*}{ Válidos } & -500 grs. & 10 & ,9 & 138 & ,2 \\
\hline & $500-749$ & 147 & 12,6 & 5738 & 9,1 \\
\hline & $750-999$ & 316 & 27,0 & 11064 & 17,5 \\
\hline & $1000-1249$ & 381 & 32,6 & 18370 & 29,0 \\
\hline & $1250-1500$ & 316 & 27,0 & 28015 & 44,2 \\
\hline & Total & 1170 & 100,0 & 63325 & 100,0 \\
\hline Perdidos & Sistema & 32 & & & \\
\hline \multicolumn{2}{|l|}{ Total } & 1202 & 100,0 & 63325 & 100,0 \\
\hline
\end{tabular}


2. Datos de la encuesta antes de la ponderación

\begin{tabular}{|l|l|c|c|}
\hline \multicolumn{2}{|c|}{ ESTADÍSTICOS DE PESO Y AÑO DE NACIMIENTO } \\
\hline \multirow{2}{*}{ N } & Válido & Peso (en gramos) & Año de nacimiento \\
\cline { 2 - 4 } & Perdidos & 1170 & 1202 \\
\hline Media & 32 & 0 \\
\hline Desviación estándar & 1049,56 & 2005,29 \\
\hline Coeficiente de variación & 265,965 & 5,176 \\
\hline
\end{tabular}

\begin{tabular}{|c|c|c|c|c|c|}
\hline \multicolumn{6}{|c|}{ FRECUENCIAS Y PORCENTAJES POR AÑO DE NACIMIENTO } \\
\hline & & Frecuencia & Porcentaje & Porcentaje válido & Porcentaje acumulado \\
\hline \multirow{20}{*}{ Válido } & 1993 & 29 & 2,4 & 2,4 & 2,4 \\
\hline & 1994 & 23 & 1,9 & 1,9 & 4,3 \\
\hline & 1995 & 22 & 1,8 & 1,8 & 6,2 \\
\hline & 1996 & 23 & 1,9 & 1,9 & 8,1 \\
\hline & 1997 & 20 & 1,7 & 1,7 & 9,7 \\
\hline & 1998 & 34 & 2,8 & 2,8 & 12,6 \\
\hline & 1999 & 33 & 2,7 & 2,7 & 15,3 \\
\hline & 2000 & 53 & 4,4 & 4,4 & 19,7 \\
\hline & 2001 & 48 & 4,0 & 4,0 & 23,7 \\
\hline & 2002 & 72 & 6,0 & 6,0 & 29,7 \\
\hline & 2003 & 64 & 5,3 & 5,3 & 35,0 \\
\hline & 2004 & 73 & 6,1 & 6,1 & 41,1 \\
\hline & 2005 & 57 & 4,7 & 4,7 & 45,8 \\
\hline & 2006 & 38 & 3,2 & 3,2 & 49,0 \\
\hline & 2007 & 66 & 5,5 & 5,5 & 54,5 \\
\hline & 2008 & 98 & 8,2 & 8,2 & 62,6 \\
\hline & 2009 & 88 & 7,3 & 7,3 & 70,0 \\
\hline & 2010 & 140 & 11,6 & 11,6 & 81,6 \\
\hline & 2011 & 221 & 18,4 & 18,4 & 100,0 \\
\hline & Total & 1202 & 100,0 & 100,0 & \\
\hline
\end{tabular}


3. Datos de la encuesta tras la ponderación

\begin{tabular}{|l|l|c|c|}
\hline \multicolumn{3}{|c|}{ ESTADísticos DE PESO Y AÑO DE NACIMIENTO } \\
\hline \multirow{2}{*}{$N$} & Peso (en gramos) & Año de nacimiento \\
\hline & Válido & 1158 & 1190 \\
\cline { 2 - 4 } & Perdidos & 32 & 0 \\
\hline Media & 1152,20 & 2003,70 \\
\hline Desviación estándar & 263,267 & 5,255 \\
\hline Coeficiente de variación & $22,85 \%$ & - \\
\hline
\end{tabular}

\begin{tabular}{|c|c|c|c|c|c|}
\hline \multicolumn{6}{|c|}{ FRECUENCIAS Y PORCENTAJES POR AÑO DE NACIMIENTO } \\
\hline & & Frecuencia & Porcentaje & Porcentaje válido & Porcentaje acumulado \\
\hline \multirow{20}{*}{ Válido } & 1993 & 35 & 2,9 & 2,9 & 2,9 \\
\hline & 1994 & 37 & 3,1 & 3,1 & 6,0 \\
\hline & 1995 & 37 & 3,1 & 3,1 & 9,1 \\
\hline & 1996 & 45 & 3,8 & 3,8 & 12,8 \\
\hline & 1997 & 44 & 3,7 & 3,7 & 16,5 \\
\hline & 1998 & 44 & 3,7 & 3,7 & 20,2 \\
\hline & 1999 & 51 & 4,3 & 4,3 & 24,4 \\
\hline & 2000 & 54 & 4,5 & 4,5 & 29,0 \\
\hline & 2001 & 57 & 4,8 & 4,8 & 33,7 \\
\hline & 2002 & 65 & 5,4 & 5,4 & 39,2 \\
\hline & 2003 & 62 & 5,2 & 5,2 & 44,4 \\
\hline & 2004 & 69 & 5,8 & 5,8 & 50,2 \\
\hline & 2005 & 71 & 5,9 & 5,9 & 56,2 \\
\hline & 2006 & 72 & 6,1 & 6,1 & 62,2 \\
\hline & 2007 & 82 & 6,9 & 6,9 & 69,1 \\
\hline & 2008 & 95 & 8,0 & 8,0 & 77,1 \\
\hline & 2009 & 92 & 7,8 & 7,8 & 84,9 \\
\hline & 2010 & 90 & 7,6 & 7,6 & 92,4 \\
\hline & 2011 & 90 & 7,6 & 7,6 & 100,0 \\
\hline & Total & 1190 & 100,0 & 100,0 & \\
\hline
\end{tabular}

